\title{
Patient-reported impact of Charcot-Marie-Tooth disease: protocol for a real-world digital lifestyle study
}

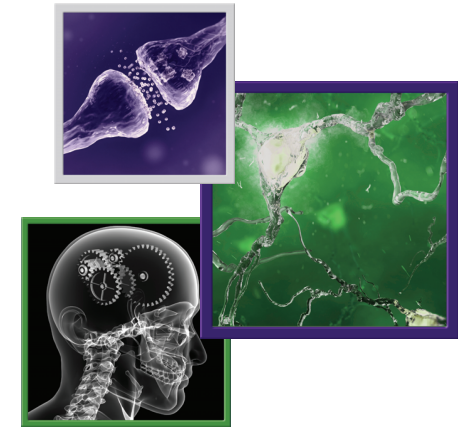

\author{
Florian P Thomas¹, Mario Saporta², Shahram Attarian³ (iD, Teresa Sevilla4 (iD, Rafael Sivera \\ Mascaró $^{5}$, Gian Maria Fabrizi ${ }^{6}$, Filippo Genovese 7 , Amy Gray ${ }^{8}$, Simon Bull ${ }^{9}$, Daniel \\ Tanesse $^{10}$, Manuel Rego ${ }^{11}$, Allison Moore ${ }^{12}$, Courtney Hollett ${ }^{12}$, Katia Monteiro ${ }^{13}$, Xavier \\ Paoli $^{13}$, Samuel Llewellyn ${ }^{14}$ (i), Mark Larkin*,14 (iD) \& Youcef Boutalbi ${ }^{13}$ \\ ${ }^{1}$ Hackensack Meridian School of Medicine, Hackensack University Medical Center, Hackensack, NJ, USA \\ ${ }^{2}$ University of Miami Miller School of Medicine, Miami, FL, USA \\ ${ }^{3} \mathrm{CHU}$ de Marseille-Hôpital de la Timone, Marseille, France \\ ${ }^{4}$ University of Valencia, Hospital Universitari i Politècnic La Fe, Valencia, Spain \\ ${ }^{5}$ Hospital Francesc de Borja, Gandia, Spain \\ ${ }^{6}$ Department of Neurosciences, Biomedicine \& Movement Sciences, University of Verona, Verona, Italy \\ ${ }^{7}$ ACMT-Rete per la malattia di Charcot-Marie-Tooth OdV, Bologna, Italy \\ ${ }^{8}$ Charcot-Marie-Tooth Association, Glenolden, PA, USA \\ ${ }^{9}$ Charcot-Marie-Tooth UK, Christchurch, UK \\ ${ }^{10} \mathrm{CMT}$ France, Saint-Alban, France \\ ${ }^{11}$ Federación Española de Enfermedades Neuromusculares, Barcelona, Spain \\ ${ }^{12}$ Hereditary Neuropathy Foundation, New York, NY, USA \\ ${ }^{13}$ Pharnext, Paris, France \\ ${ }^{14}$ Vitaccess Ltd, Oxford, UK \\ *Author for correspondence: mark.larkin@vitaccess.com
}

\section{Practice points}

- Charcot-Marie-Tooth disease (CMT) is a rare, chronic, progressive motor and sensory neuropathy. Little is known about the burden it imposes on patients, their caregivers and society. There is a lack of data collected directly from people who have the condition in the real-world setting.

- The objective of this study is to provide a detailed view of the impact of CMT and its treatment on patients in the real-world setting, including factors such as epidemiology, natural history and clinical and humanistic burden.

- This is a prospective, real-world, patient-reported lifestyle study. Adults with CMT in France, Germany, Italy, Spain, the UK and the USA will use a smartphone app, CMT\&Me, to enter regular data about CMT, its management and its impact on their lives over a period of at least 2 years.

- Approximately 2000 participants will be enrolled using community-based recruitment methods, mostly through patient advocacy group networks.

- Shortly after enrollment, participants will be asked to complete a profile that includes data on demographics, lifestyle characteristics, diagnosis and treatments.

- Participants will be asked to complete a number of patient-reported outcome instruments - after enrollment and then either monthly (EuroQol 5 Dimensions 5 Levels, Brief Fatigue Inventory, Patient-Reported Outcomes Measurement Information System Pain Intensity 3a and Interference 6b, bespoke questionnaires for the study, Patient-Reported Outcomes Measurement Information System Sleep Disturbance 8a) or quarterly (Work Limitations Questionnaire, Falls Efficacy Scale-International, Lower Extremity Function Scale, Quick Disabilities of Arm, Shoulder \& Hand) for up to 2 years - that assess health-related quality of life, specific symptoms and function.

- Interim analyses will be conducted upon registration of the 1000th participant and at 12-month intervals from study launch. A final analysis will be presented at study end (currently 2 years from study launch).

- A scientific advisory board comprised of independent clinical and patient advocacy group representatives from each study country plus sponsor and Vitaccess representatives was convened during the study design phase to protect participants' interests.

- A publication plan for the study includes conference presentations and journal publications. Study progress and results will be communicated to participants on an ongoing basis through regular email, social media and in-app communication. 
Charcot-Marie-Tooth disease (CMT) is a rare, chronic, progressive motor and sensory neuropathy affecting the peripheral nervous system. This study will explore the real-world impact of CMT. The trial is a digital study of approximately 2000 people in 6 countries with CMT $\geq 18$ years. Participants will use a smartphone application to check eligibility, provide consent and contribute data. The dataset will include a personal profile, covering demographics, lifestyle, diagnosis and treatment and a selection of validated generic and disease-specific instruments. Participants will provide data for up to 2 years. Data analysis will be conducted upon registration of the 1000th participant and at 12-month intervals from launch. This study is designed to help researchers and clinicians understand the real-world impact of CMT and the unmet needs of patients.

ClinicalTrials.gov identifier: NCT03782883

Lay abstract: Charcot-Marie-Tooth disease (CMT) is a rare condition causing weakness of the limbs that worsens gradually over time. This study will explore the impact of CMT on patients and caregivers. This is a digital study of approximately 2000 adults in 6 countries with CMT. Participants will use a smartphone application to provide their consent and contribute data. Data will be collected via a personal profile, covering demographics, lifestyle, diagnosis and treatment and a selection of generic and disease-specific surveys. Data analysis will be conducted upon registration of the 1000th participant and at 12-month intervals from launch. This study is designed to help researchers and clinicians understand the impact of CMT and the unmet needs of patients.

First draft submitted: 11 August 2020; Accepted for publication: 1 October 2020; Published online: 22 October 2020

Keywords: burden of illness $\bullet$ Charcot-Marie-Tooth disease $\bullet$ international $\bullet$ observational $\bullet$ patient-reported outcomes

Charcot-Marie-Tooth disease (CMT) is a rare, chronic, progressive motor and sensory neuropathy that affects the peripheral nervous system, leading to progressive, predominantly distal, muscle weakness, atrophy, sensory loss and progressive limb dysfunction [1]. With an estimated prevalence of 1 in 2500 people, it is the most frequently inherited neuropathy and one of the most common neurogenetic disorders [2].

CMT compromises patient lifestyles, everyday activities, career and family choices [3]. There is currently no cure for CMT [4]. Treatment focuses on physical therapy to maintain movement, muscle strength and flexibility, combined with occupational therapy, orthotics, pain management and psychological and social support [5]. Surgical intervention may be required for more severe forms of the disease.

\section{Background \& rationale}

As with many other rare conditions, there is a lack of data about the burden of CMT with which to make informed decisions to improve disease management and outcomes. In particular, little evidence has been collected directly from people who have the condition, nor has much data been gathered in the real-world setting.

Patient reports often provide unique perspectives and in-depth depictions of disease burden and the impact of treatment. Thus, the role of patient-reported outcome (PRO) data is becoming increasingly recognized in strengthening disease understanding and in the development, regulatory approval, use and reimbursement of treatments. To date, much PRO evidence has been generated in the clinical trial setting, with little data collected from patients being managed in real-world clinical practice. Real-world evidence (RWE) can provide more granular, longer-term data from a broader patient population than is typical in clinical trials, so there is clear value in its collection and analysis. In rare diseases like CMT, where there are likely to be challenges in conducting adequately sized and controlled clinical trials, RWE is of particular worth.

A growing recognition that RWE has a complementary value to randomized controlled trial evidence has been matched in recent years by regulators and health technology assessment agencies publishing guidance for use of RWE [6-8]. RWE is valued by regulators as a basis for regulatory decision-making, including approval of new indications for licensed drugs, as seen in the 21st Century Cures Act [9] and European Medicines Agency adaptive pathways [10]. RWE is also accepted by many health technology assessment agencies (e.g., the National Institute for Health and Care Excellence and the Canadian Agency for Drugs and Technologies in Health) and payers in health technology assessment submissions, including for orphan drugs [11]. 
There is a current need for a real-world study to explore the burden of CMT, a study that collects data directly from patients about their experiences of living with - and managing - their condition. The objective of this study, which is currently in the data collection phase, is to provide a detailed view of the impact of CMT and its treatment on patients in the real-world setting, including factors such as epidemiology, natural history and clinical and humanistic burden.

\section{Design}

\section{Study design}

This is a prospective, real-world, patient-reported lifestyle study. Adults with CMT use a smartphone app, CMT\&Me (Vitaccess Ltd, Oxford, UK), to enter regular data about CMT, its management and its impact on their lives over a period of at least 2 years.

\section{Study setting}

Participation is entirely via the CMT\&Me app. There are no physical study sites. Data are collected from participants in the following countries: France, Germany, Italy, Spain, the UK and the USA.

\section{Eligibility criteria}

Inclusion criteria are as follows:

- Adult (age $\geq 18$ years) diagnosed with CMT (self-reported diagnosis only);

- Resident in one of the study countries;

- Willing to use their own smartphone/tablet;

- Willing to provide informed consent.

There are no specific exclusion criteria.

\section{Informed consent}

Participants enroll and provide informed consent via the CMT\&Me app. Participant briefing materials are presented in a series of pages on the app, each followed by an informed consent statement relating to that section. Participants who agree to all the statements are considered to have given informed consent. Consent includes agreement to the possibility of data being cross-referenced with other medical databases.

\section{Risks}

As this is a non-interventional study, participants are not expected to be at risk of physical harm. Participation may trigger negative feelings in some participants. Participants are able to contact the research team if they have concerns or questions and are advised to contact their medical team where necessary.

\section{Outcomes}

As the aim of the study is to provide a detailed view of the impact of CMT on patients in the real-world setting, the authors are aiming to collect as much data as possible. Outcomes are as follows:

- Demographics

- Date of birth

- Height and weight (to determine body mass index)

$\circ$ Sex

- Home postal code

- Healthcare system identifier (e.g., National Health Service number) [outcome collected only in countries where permitted]

- Smoking status (“Do you smoke?" plus follow-up question to those answering yes to quantify how often the participant smokes)

- Exercise status ("How often do you take this type of exercise [at least 20 minutes]?")

- Diet status (the participant's regular diet from a list of categories) 
- Alcohol consumption status (how many units the participant drinks per day, factoring in differences in unit worth across countries)

- CMT characteristics

- CMT subtype

- Comorbidities

- CMT diagnosis and treatment

- $\quad$ Age at symptom onset

- Age when medical care was first sought

- Age at diagnosis

- Diagnostic tests performed to achieve CMT diagnosis

- Clinical examinations performed to understand CMT diagnosis

- Physical therapies received for CMT

- Medicines received for CMT

- Walking aids/orthotics received for CMT

- Type of medical professionals seen for CMT

- Frequency of visits to the emergency department

- CMT symptoms

- Symptoms experienced

- Brief Fatigue Inventory (BFI)

- $\quad$ Patient-Reported Outcomes Measurement Information System (PROMIS ${ }^{T M}$ ) Pain Intensity 3a

- $\quad$ PROMIS ${ }^{T M}$ Pain Interference 6b

- Cramp frequency

- Cramp intensity

- Work/study

- Work Limitations Questionnaire (WLQ)

- Work/study status

- Days of work missed due to CMT

- Health-related quality of life (HRQoL)

- EuroQol 5 Dimensions 5 Levels (EQ-5D-5L)

- Physical function

- $\quad$ PROMIS ${ }^{T M}$ Sleep Disturbance 8a

- $\quad$ Falls Efficacy Scale - International (FES-I)

- Lower Extremity Function Scale (LEFS)

- Quick Disabilities of Arm, Shoulder \& Hand (QuickDASH)

Planned sample size

The authors aim to enroll approximately 2000 participants. A formal sample size calculation has not been performed, as hypothesis testing is not planned and analysis is descriptive in nature.

\section{Recruitment}

Recruitment is community-based, with potential participants made aware of the study via direct communication from CMT patient advocacy groups (PAGs), PAG and Vitaccess Ltd (CMT\&Me app developer and study contract research organization) social media accounts and word of mouth, including via PAG community networks and patient ambassadors. 


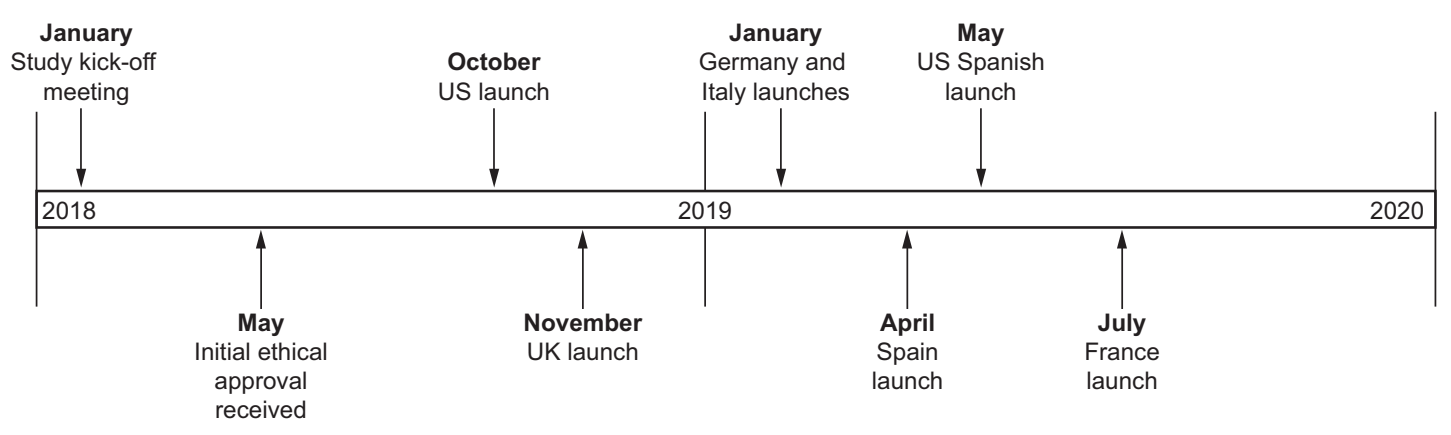

Figure 1. Study timeline.

Would-be participants are able to download the CMT\&Me app from Apple's App Store or Google Play, but study registration is contingent on meeting eligibility criteria.

The app was launched sequentially across the study countries in the following stages:

- Stage 1

- $\quad$ US English (October 15, 2018)

- UK English (November 9, 2018)

- Stage 2

- $\quad$ Germany (January 31, 2019)

○ Italy (January 31, 2019)

- Stage 3

- $\quad$ Spain (April 8, 2019)

- US Spanish (May 8, 2019)

- Stage 4

- $\quad$ France (July 1, 2019)

Figure 1 summarizes the study timeline. Figure 2 shows select example screenshots from the CMT\&Me app.

\section{Data collection \& management}

Shortly after enrollment, participants are asked to complete a profile, which includes data on demographics, lifestyle characteristics, diagnosis and treatments, many of which are expected to remain fairly stable over the duration of the study. For those data that may change over the duration of the study (e.g., treatments, healthcare visits), participants are able - and encouraged - to add, edit or remove them.

Participants are also asked to complete a number of PRO instruments - after enrollment and then either monthly (EQ-5D-5L, BFI, PROMIS ${ }^{\text {TM }}$ Pain Intensity 3a and Interference 6b, bespoke questionnaires for the study, PROMIS $^{T M}$ Sleep Disturbance 8a) or quarterly (WLQ, FES-I, LEFS, QuickDASH) - that assess HRQoL, specific symptoms and function for up to 2 years. Summary descriptions of all PRO instruments are provided below. Figure 3 summarizes the participant timeline.

$E Q-5 D-5 L$

The EQ-5D-5L comprises two parts: the EQ-5D-5L descriptive system and the EQ Visual Analogue Scale [12]. The descriptive system comprises five dimensions (mobility, self-care, usual activities, pain/discomfort and anxiety/depression), each with five levels (no problems, slight problems, moderate problems, severe problems 


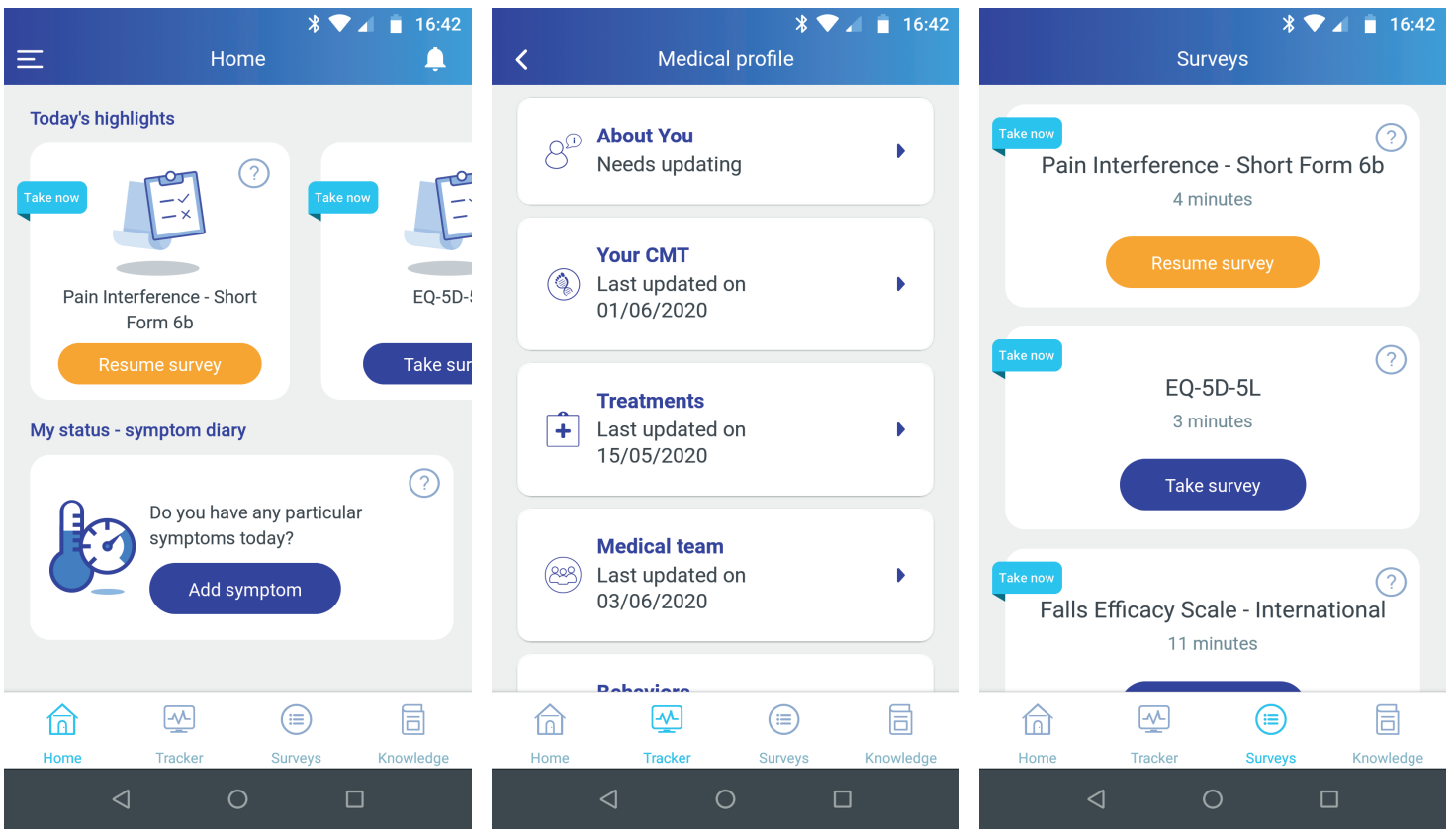

Figure 2. Select screenshots from the CMT\&Me app.

Reproduced with permission from Vitaccess Ltd.

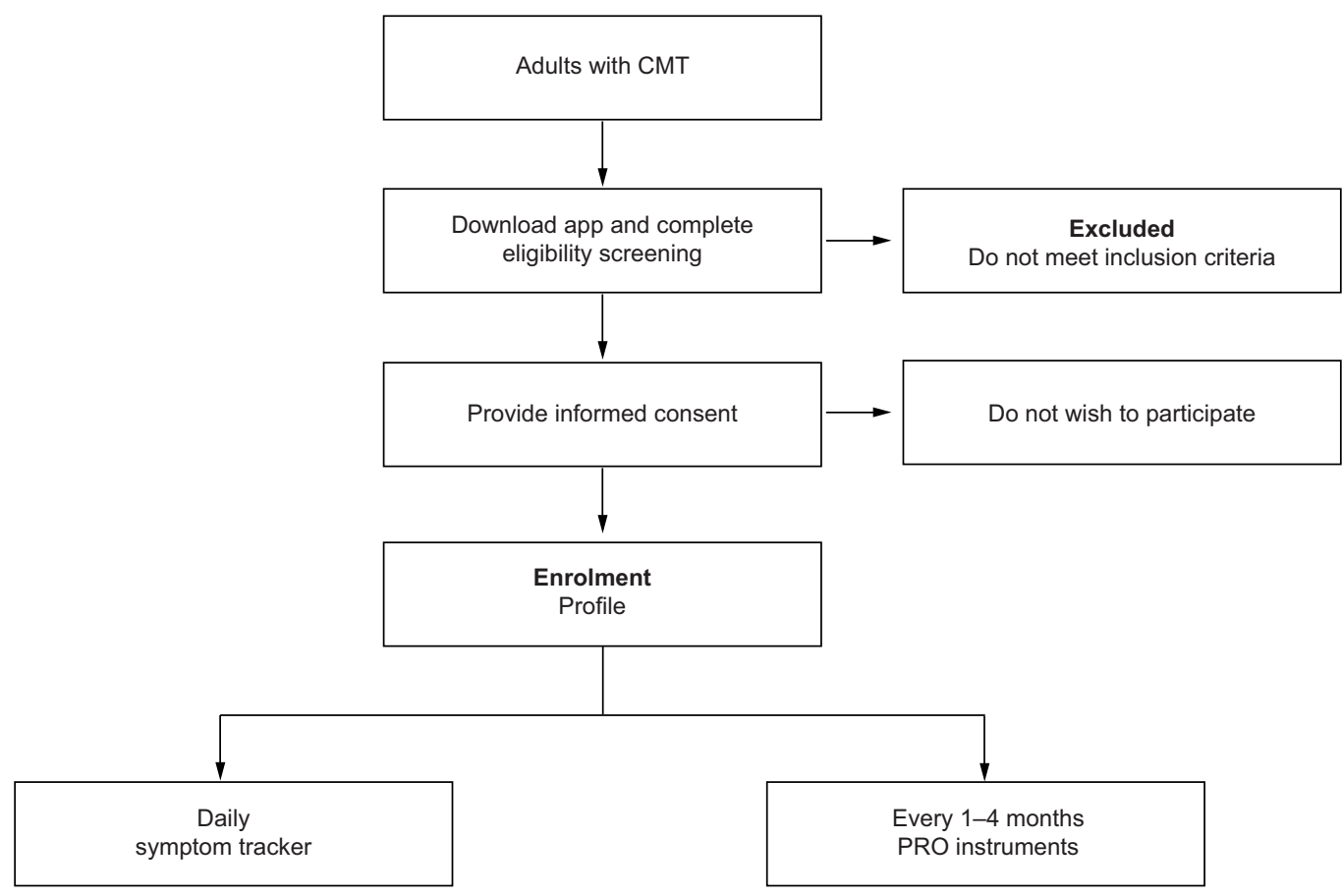

Figure 3. Participant timeline.

CMT: Charcot-Marie-Tooth disease; PRO: Patient-reported outcome. 
and extreme problems - i.e., higher scores represent worse health). The scores for the five dimensions are combined in a 5-digit number describing the participant's health state.

The EQ Visual Analogue Scale records the participant's self-rated health on a vertical, visual analogue scale, with endpoints labeled "the best health you can imagine" and "the worst health you can imagine". Higher scores represent better self-perceived health.

\section{$B F I$}

The BFI assesses participants' fatigue severity [13]. The measure uses a 10-point numeric rating scale and a recall period of 24 hours. A global fatigue score is calculated by averaging all 9 items.

\section{PROMIS ${ }^{\mathrm{TM}}$ Pain Intensity $3 a$ \& Interference $6 b$}

The PROMIS ${ }^{T M}$ Pain Intensity $3 \mathrm{a}$ includes two items that assess pain intensity over the last 7 days (average and worst pain) and one item that assesses pain intensity "right now", each score using a 5-point scale [14]. Possible scores range from 2 to 10, where higher scores represent worse pain. This measure is generic rather than disease-specific.

The PROMIS Pain Interference $6 \mathrm{~b}$ assesses the extent to which pain hinders engagement with social, cognitive, physical and recreational activities as well as enjoyment in life over the last 7 days using a 5-point scale [15]. Possible scores range from 6 to 30, where higher scores represent greater interference. This measure is generic rather than disease-specific.

\section{Bespoke questionnaires for this study}

Two cramp-specific items measuring cramp frequency and intensity were developed for inclusion in the study. The cramp frequency item asks, "In the past 7 days, how many days did you experience cramp?" and has 5 possible response options: had no cramp, 1-2 days, 3-4 days, 5-6 days, every day. The cramp intensity item asks, "In the past 7 days, how intense was your cramp at its worst?" and has 5 possible response options: had no cramp, mild, moderate, severe, very severe. Higher scores on both items represent greater cramp frequency and intensity, respectively.

\section{WLQ}

The WLQ measures the impact of CMT on participants' work ability and productivity across 4 domains: time management, physical demands, mental-interpersonal demands and output demands [16]. Possible domain scores range from 0 to 100 , and the recall period is the previous 2 weeks. WLQ domain scores will be converted into an estimate of productivity loss using an algorithm.

\section{PROMIS ${ }^{\mathrm{TM}}$ Sleep Disturbance 8 a}

The PROMIS ${ }^{T M}$ Sleep Disturbance 8 a assesses self-reported perceptions of sleep quality, sleep depth and restoration associated with sleep over the last 7 days using a 5-point scale [17]. Possible scores range from 8 to 40, where higher scores represent worse sleep disturbance. This measure is generic rather than disease-specific.

\section{FES-I}

The FES-I measures the level of concern about falling during social and physical activities inside and outside the home, whether or not the person actually carries out the activity [18]. The 'usual' level of concern is measured on a 4-point Likert scale ( $1=$ not at all concerned; 4 = very concerned), with participants asked to consider how they usually carry out the activity. Possible scores range from 16 to 64, where higher scores represent greater concern about falling.

\section{LEFS}

The LEFS evaluates difficulties because of lower limb problems in 20 activities, including work/school activities, hobbies, moving around the home, dressing, lifting, standing, sitting, walking and running [19]. The level of difficulty is assessed for 'today' using a 5-point Likert scale $(0=$ extreme difficulty or unable to perform activity; $5=$ no difficulty). Lower scores represent greater difficulties experienced because of lower limb problems.

\section{QuickDASH}

The QuickDASH measure uses 11 items to gauge physical function and symptoms in people with any or multiple musculoskeletal disorders of the upper limbs [20]. All questions are rated 1-5 (no difficulty/none/not at all to 
unable/extreme difficulty). Possible scores range from 11 to 55, where higher scores represent greater difficulties with physical function and symptoms.

\section{Plans to promote participant retention \& complete follow-up}

If at any study time point participants do not complete data entry for a certain section of their profile or a PRO instrument within a 1-week window, their data will be considered missing for that time point. They will still be able to enter data again at later required time points.

To promote engagement with the study app and continued data entry, participants will receive in-app messages, encouraging them to complete required data entry, thanking them for doing so and stressing the importance of their contributions to research. Participants will also receive information about the study, updates on its progression and emerging results via the study app, social media and regular email newsletters.

CMT\&Me also contains non-study features that are designed to help participants learn about or track their condition. These include a symptom tracker (which allows participants to record the severity of different symptoms at any given time and create a PDF diary that can be downloaded for personal use) and knowledge articles (multimedia information on the disease and treatments based on validated information provided by PAGs). The collection of biometric data (e.g., heart rate, steps taken) via the study app and/or linkage to other apps/devices has also been explored and is feasible; however, nothing has been implemented at present. If it appears that these data would be valuable, collection will be implemented subject to ethical approvals.

\section{Data management}

To promote data quality, rules were set for question responses (e.g., range limits for continuous variables, minimization of free-text data fields, limits to number of response options that can be selected). Data will also be checked and cleaned before analysis. Limits for continuous variables were determined to constrain erroneous data but still allow sufficient variation (e.g., height of 8 feet would be prevented, but 7 feet would be permitted). The minimization of free-text fields was intended to try to systematize responses to keep completion of the surveys relatively fast for participants; the same was true for limiting response options where possible. These were part of the registry's testing and confirmed/refined as part of that process.

Each participant will log in to CMT\&Me using unique self-generated login credentials, which are unknown and inaccessible to the study team. Data saved by participants in CMT\&Me will be transferred to a central database and then aggregated and de-identified as soon as is practicable. No personal data will be held on participants' devices. Personally identifiable information will be encrypted with unique encryption keys at rest, and all data will be encrypted in transit. All study data will be stored on a secure server.

\section{Confidentiality}

All personally identifiable information will be protected by industry-standard methods, ensuring full confidentiality is maintained. Personally identifiable information is not held on participants' devices and cannot be viewed by the study sponsor or any external researchers who apply for access to the study data. Data will be stored in a central database in aggregated, de-identified form.

\section{Statistical methods \\ Statistical methods for primary \& secondary outcomes}

Data management and analysis will follow a predefined statistical analysis plan. As this is an exploratory observational study, differences and patterns in the data will be analyzed, but without exploring causation. All analyses will be descriptive, and no hypotheses will be tested. Aggregated, de-identified data will be summarized as follows:

- For continuous variables, distributions: number, mean, standard deviation, median, minimum, maximum, 95\% CI;

- For categorical variables, summaries: n, frequency, proportion.

For both variable types, the number and proportion of missing data will also be reported. Descriptive distribution statistics for each PRO instrument score, or domain score, will be presented for baseline (first data entry time point) and at each time point thereafter, including at study end. Distribution statistics will also be generated to describe the outcomes at each time point for the absolute value and the calculated change from baseline. 


\section{Interim analyses}

Interim analyses will be conducted upon registration of the 1000th participant and at 12-month intervals from study launch. The aim of the interim analyses is to inform the progression of key study outcomes, whether changes need to be made to existing survey structure and whether further exploratory analyses outside of what is detailed in the statistical analysis plan will be conducted. A final analysis will be presented at study end (currently 2 years from study launch) based on data from all participants who have completed at least one PRO instrument and the necessary elements of their profile and have been enrolled in the study.

Methods in analysis to handle protocol nonadherence \& any statistical methods to handle missing data

Missing data will be handled as set out in the scoring guidelines for the PRO instruments and according to best practice for the profile. All missing data will be assumed to be missing at random, and no adjustments will be made to account for missing data.

Plans to give access to the full protocol, participant-level data \& statistical code

Members of the public and external researchers can apply via the study website to be granted access to aggregated and anonymized study data. Access decisions will be granted under the purview of the study scientific advisory board (SAB).

\section{Oversight \& monitoring}

A key tenet of governance and monitoring is that the framework is transparent and public and so accessible to would-be participants considering enrolling as well as to enrolled participants. As such, all oversight and monitoring arrangements will be published on publicly available study web pages (https://vitaccess.com/cmt-and-me).

\section{Composition of the data monitoring committee, its role \& reporting structure}

An SAB was convened during the study design phase to protect participants' interests. The SAB is responsible for the following:

- Effective operational management of the study;

- Ensuring that the study operates in the best interests of participants;

- Ensuring that the study operates to the highest levels of academic rigor.

The SAB acts as an advisory/review body for the following:

- Publication strategy and publications;

- Data analyses;

- Study evolution: possible amendments to the study protocol;

- Communication: input into materials and communication with the participant cohort and wider CMT community.

The SAB also acts as a decision-making body for third-party data access requests. In line with good practice recommendations [21], the SAB comprises independent clinical and PAG representatives from each study country plus sponsor and Vitaccess representatives. The SAB will meet in person at least annually (where feasible) and by teleconference at least every 6 months.

\section{Adverse event reporting \& harms}

There is no obligation to report adverse events recorded by participants. Participants who report receiving the sponsor's product, PXT3003 (or other experimental drugs), would have already been enrolled in clinical trials, and it is assumed they would therefore be followed up under the relevant adverse event reporting pathways.

Frequency \& plans for auditing study conduct

The SAB will audit the study conduct on an ongoing basis. 


\section{Plans for communicating important protocol amendments to relevant parties}

The study team will be responsible for communicating protocol amendments to relevant parties as necessary, and this must be approved by the SAB.

\section{Dissemination plans}

The sponsor and study team have developed a publication plan for the study, which was approved by the SAB, to include conference presentations and journal publications. Study progress and results will be communicated to participants on an ongoing basis through regular email, social media and in-app communication (newsletters and data nuggets).

\section{Conclusion}

CMT is a rare, chronic disease, and little is known about the burden it imposes on patients, their caregivers and society. There is a lack of data collected in the real-world setting directly from people who have the condition. Digital tools that enable real-time data reporting open up possibilities of publishing and acting upon the data faster, which is important to those affected by the disease. These tools are also useful for capturing data during periods of reduced patient access to clinics (e.g., the coronavirus disease 2019 pandemic).

This international, longitudinal, real-world digital PRO study explores the burden of CMT experienced by patients. The study provides a detailed view of the impact of CMT and its treatment on patients in the real-world setting, including factors such as epidemiology, natural history and humanistic burden. Close and collaborative partnerships with CMT PAGs, who are 'experts by experience,' will not only aid participant recruitment but will also ensure true patient centricity of the research.

As requesting clinical confirmation of diagnosis is either onerous or possible to circumvent, participants are eligible for this study based on self-reported CMT diagnosis alone. Although this means responses to questions within the study are therefore subject to error because of misinformation bias, data will be monitored on an ongoing basis - and filtered accordingly - for mitigation of outliers. Linkage studies (i.e., with existing registries of known CMT patients) also offer a powerful way of addressing the potential for error, and the informed consent includes an opt-in to facilitate such studies. The risk of recruiting false patients in the first place is very limited, however, given that the main method of recruitment is through CMT PAG networks. In conclusion, this international, longitudinal, real-world digital PRO study - the first of its kind - will undoubtedly help researchers and clinicians understand the real-world impact of CMT and the unmet needs of patients.

\section{Author contributions}

S Llewellyn contributed to conception and design of the study and wrote the first draft of the manuscript. K Monteiro, X Paoli, M Larkin and Y Boutalbi conceived and designed the study and drafted the manuscript. F P Thomas, M Saporta, S Attarian, T Sevilla, R S Mascaró, G M Fabrizi, F Genovese, A Gray, S Bull, D Tanesse, M Rego, A Moore and C Hollett contributed to conception and design of the study and reviewed the manuscript. All authors contributed to the review of the manuscript and approved the final version.

\section{Acknowledgments}

The authors would like to acknowledge the contributions of all participants who are contributing data to the study, ACMT-Rete per la malattia di Charcot-Marie-Tooth OdV (Bologna, Italy), Charcot-Marie-Tooth Association (Glenolden, PA, USA), Charcot-MarieTooth UK (Christchurch, UK), CMT France (Saint-Alban, France), Deutschen Gesellschaft für Muskelkranke e.V. (Freiburg, Germany), European Charcot-Marie-Tooth Federation (Brussels, Belgium), Federación Española de Enfermedades Neuromusculares (Barcelona, Spain), Hereditary Neuropathy Foundation (New York, NY, USA), Annabel Nixon at Chilli Consultancy (Salisbury, UK) for her work on the development of the CMT\&Me app and study design and Vitaccess staff members for their work on the development of the CMT\&Me app, study design and implementation.

Financial \& competing interests disclosure

Development of this manuscript and the study outlined within were funded by Pharnext, Paris, France. The authors declare the following conflicts of interest in relation to this article: F P Thomas is an employee of Hackensack Meridian School of Medicine, Hackensack University Medical Center. M Saporta is an employee of University of Miami Miller School of Medicine. S Attarian is an employee of CHU de Marseille-Hôpital de la Timone. T Sevilla is an employee of University of Valencia, Hospital Universitari i Politècnic La Fe. R S Mascaró is an employee of Hospital Francesc de Borja. G M Fabrizi is an employee of University of Verona. F 
Genovese is a volunteer at ACMT-Rete per la malattia di Charcot-Marie-Tooth OdV. A Gray is an employee of Charcot-Marie-Tooth Association. S Bull is an employee of Charcot-Marie-Tooth UK. D Tanesse is a volunteer at CMT France. M Rego is a volunteer for Federación Española de Enfermedades Neuromusculares. A Moore and C Hollett are employees of Hereditary Neuropathy Foundation. X Paoli and Y Boutalbi are employees of Pharnext. K Monteiro was an employee of Pharnext when the article was developed. S Llewellyn and M Larkin are employees of Vitaccess Ltd. The authors have no other relevant affiliations or financial involvement with any organization or entity with a financial interest in or financial conflict with the subject matter or materials discussed in the manuscript apart from those disclosed.

No writing assistance was utilized in the production of this manuscript.

Ethical conduct of research

Institutional review board approval (protocol number 5101-03-2018; Salus IRB, Austin, TX, USA) was obtained for conducting the study in France, Germany, Italy, the UK and the USA. Local ethics approval from El Comité de Ética de la Investigación con medicamentos del Hospital Universitario y Politécnico La Fe (protocol code PHA-CMT-2018-01; Valencia, Spain) was obtained for conducting the study in Spain.

Informed consent was obtained from all participants.

Data sharing statement

Members of the public and external researchers can apply via the study website (https://vitaccess.com/cmt-and-me) to be granted access to aggregated and anonymized study data. Access decisions will be granted under the purview of the study scientific advisory board.

\section{Open access}

This work is licensed under the Attribution-NonCommercial-NoDerivatives 4.0 Unported License. To view a copy of this license, visit http://creativecommons.org/licenses/cc-by-nc-nd/4.0/

\section{References}

Papers of special note have been highlighted as: $\bullet$ of interest; $\bullet \bullet$ of considerable interest

1. Reilly MM, Shy ME. Diagnosis and new treatments in genetic neuropathies. J. Neurol. Neurosurg. Psychiatry 80(12), 1304-1314 (2009).

- Outlines the classification of different genetic neuropathies, including Charcot-Marie-Tooth disease (CMT), and treatment options.

2. Kedlaya D. Charcot-Marie-Tooth Disease. (2020) https://emedicine.medscape.com/article/1232386-overview

- $\quad$ CMT review, updated in 2019, featuring latest epidemiology figures.

3. Hereditary Neuropathy Foundation. What It's Like to Live with Charcot-Marie-Tooth (CMT): The Stories of Those Who Know Best. (2020) www.hnf-cure.org/wp-content/uploads/2013/09/HNF-Living-With-CMT_singlepages-small.pdf

4. Pareyson D, Saveri P, Pisciotta C. New developments in Charcot-Marie-Tooth neuropathy and related diseases. Curr. Opin. Neurol. 30(5), 471-480 (2017).

5. Pareyson D, Marchesi C. Diagnosis, natural history, and management of Charcot-Marie-Tooth disease. Lancet Neurol. 8(7), 654-667 (2009).

- Outlines the clinical presentation of CMT, its natural history and treatment options.

6. Faria A, Hernandez Alva M, Manca A, Wailoo AJ. NICE DSU Technical Support Document 17: The use of observational data to inform estimates of treatment effectiveness for technology appraisal: methods for comparative individual patient data. (2020) https://nicedsu.org.uk/wp-content/uploads/2016/03/TSD17-DSU-Observational-data-FINAL.pdf

7. US FDA. Framework for FDA's Real-World Evidence Program. (2020) www.fda.gov/media/120060/download

8. CADTH. Use of Real-World Evidence in Single-Drug Assessments Environmental Scan. (2020) https://www.cadth.ca/use-real-world-evidence-single-drug-assessments-environmental-scan

9. 21st Century Cures Act. Public Law 114-225, 114th Congress. (2020) www.govinfo.gov/content/pkg/PLAW-114publ255/pdf/PLAW-114publ255.pdf

10. European Medicines Agency. Final report on the adaptive pathways pilot. (2020) www.ema.europa.eu/en/documents/report/final-report-adaptive-pathways-pilot_en.pdf

11. Åkesson C, Llewellyn S, Bagshaw E, Kousoulakou H, Larkin M. Understanding the role of real-world evidence in health technology assessment for orphan drugs. Value Health 22, S862 (2019).

- Targeted review demonstrating how real-world evidence (RWE) is accepted by health technology assessment (HTA) bodies in orphan drug submissions. 
12. Herdman M, Gudex C, Lloyd A et al. Development and preliminary testing of the new five-level version of EQ-5D (EQ-5D-5L). Qual. Life Res. 20(10), 1727-1736 (2011).

13. Mendoza TR, Wang XS, Cleeland CS et al. The rapid assessment of fatigue severity in cancer patients: use of the Brief Fatigue Inventory. Cancer 85(5), 1186-1196 (1999).

14. Revicki DA, Chen W-H, Harnam N et al. Development and psychometric analysis of the PROMIS pain behavior item bank. Pain 146(1-2), 158-169 (2009).

15. Amtmann D, Cook KF, Jensen MP et al. Development of a PROMIS item bank to measure pain interference. Pain 150(1), 173-182 (2010).

16. Lerner D, Amick Iii BC, Rogers WH, Malspeis S, Bungay K, Cynn D. The Work Limitations Questionnaire. Med. Care 39(1), 72-85 (2001).

17. Yu L, Buysse DJ, Germain A et al. Development of short forms from the PROMIS ${ }^{T M}$ sleep disturbance and sleep-related impairment item banks. Behav. Sleep Med. 10(1), 6-24 (2012).

18. Yardley L, Beyer N, Hauer K, Kempen G, Piot-Ziegler C, Todd C. Development and initial validation of the Falls Efficacy Scale-International (FES-I). Age Ageing 34(6), 614-619 (2005).

19. Binkley JM, Stratford PW, Lott SA, Riddle DL. The Lower Extremity Functional Scale (LEFS): scale development, measurement properties, and clinical application. Phys. Ther. 79(4), 371-383 (1999).

20. Beaton DE, Wright JG, Katz JN, Upper Extremity Collaborative Group. Development of the QuickDASH: comparison of three item-reduction approaches. J. Bone Joint Surg. Am. 87(5), 1038-1046 (2005).

21. Berger ML, Sox H, Willke RJ et al. Good practices for real-world data studies of treatment and/or comparative effectiveness: recommendations from the joint ISPOR-ISPE Special Task Force on real-world evidence in health care decision making. Pharmacoepidemiol. Drug Saf. 26(9), 1033-1039 (2017).

•• Outlines recommendations for good procedural practices for real-world data studies such as this one. 


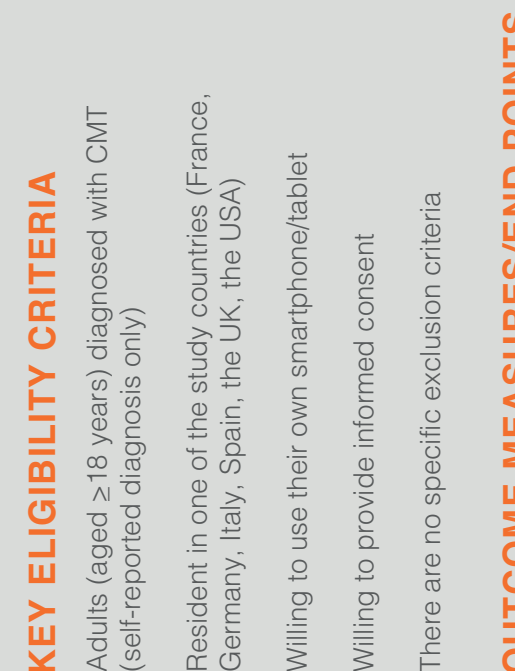

占

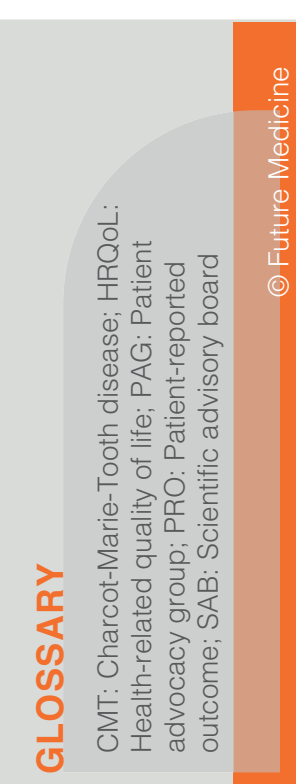

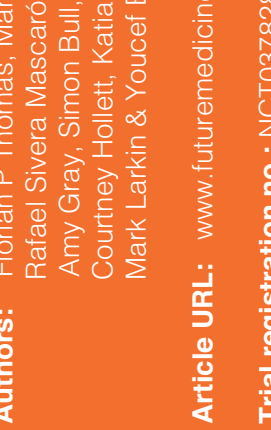
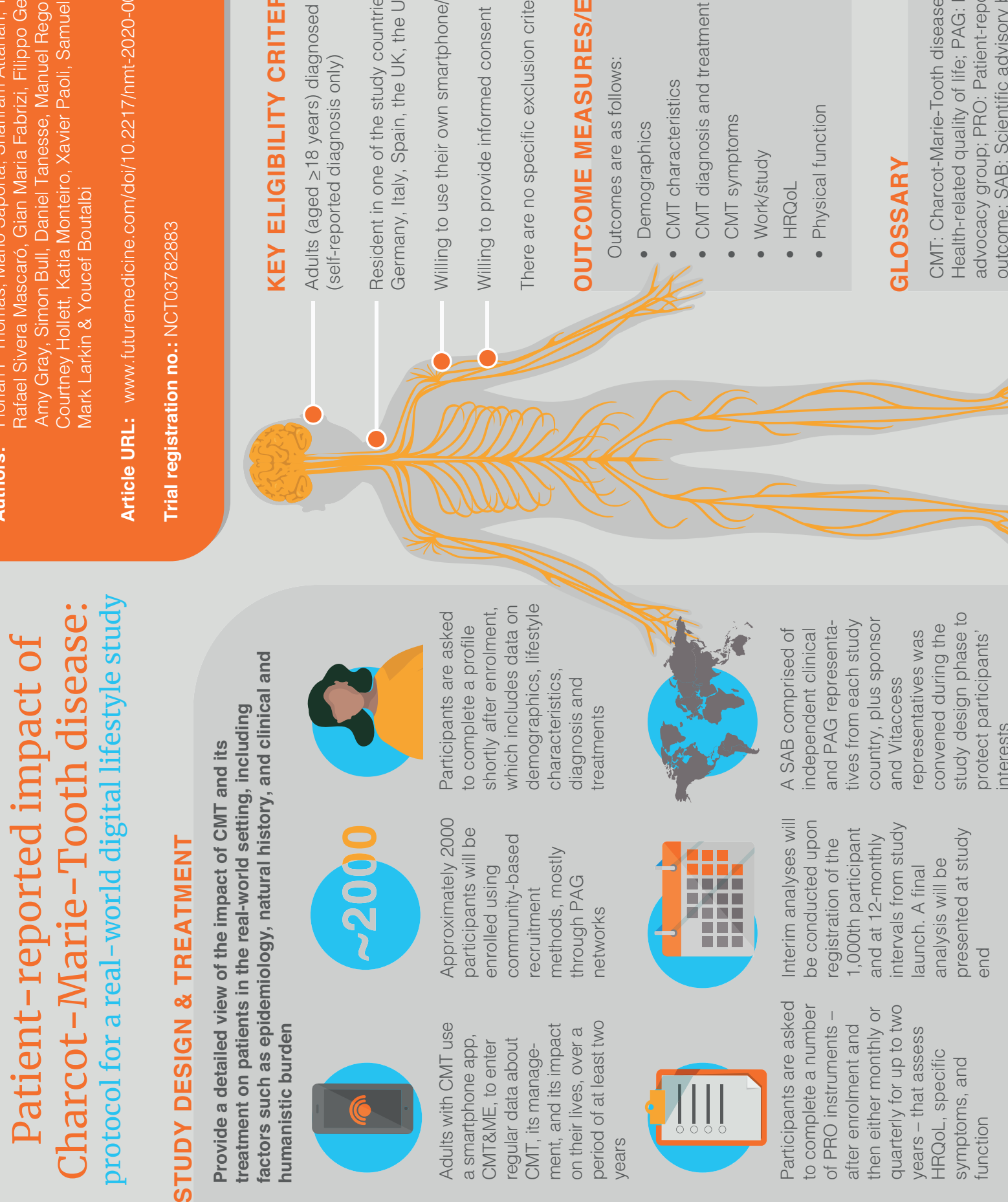

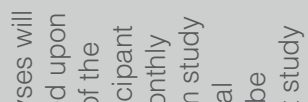

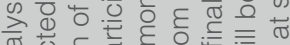

ฮ

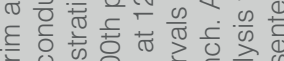

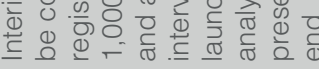

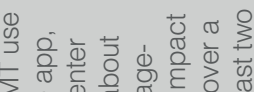

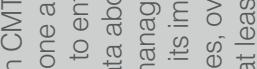

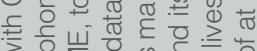

उ

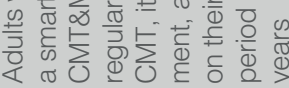

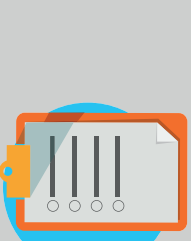

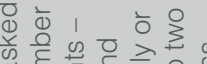

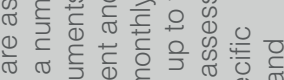

$\omega$

ब $\frac{0}{2}=\frac{0}{2}$

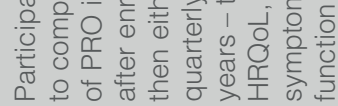

